\section{A spectral-analysis program with graphics for the CBM/PET/C-64 microcomputers}

\author{
D. JAMES CAMPBELL \\ University of Wollongong, Wollongong, \\ New South Wales, Australia
}

Biological rhythms are an important aspect of behavior (Rusak \& Zucker, 1975; Saunders, 1977). Circadian rhythms, which have a periodicity of approximately $24 \mathrm{~h}$, have received most attention, but short- and long-term rhythms also play a role in behavior (Broom, 1979, 1980; Campbell \& Shipp, 1974, 1979; Daan \& Slopsema, 1978). Various methods for detecting such rhythms have been described (Broom, 1979; Campbell \& Schipp, 1974; Linkens, 1979; Westman, 1977). Spectral analysis is a technique that is particularly useful for this purpose, and examples of its application are those of Broom (1979); Campbell and Shipp (1974). A spectral-analysis tutorial was provided by Sturgis (1983).

The program described here can be used for the analysis of data collected by an activity monitor wired to a datalogging system such as those described by Campbell and Forest (1984) and Martin and Unwin (1980) or of any body of data collected at equal time intervals (unit time intervals). Long records are desirable (say, $>100$ ), particularly if the record is noisy. The program has been written to operate on the CBM/PET/C-64 microcomputers with all versions of BASIC in conjunction with the various Commodore peripherals. Input can be via keyboard, tape, or disk. Output can be to the screen or printer and can be saved on tape or disk. The graphics display and input/output routines are essentially the same as those used in the data editor and high-resolution histogram plotter described by Campbell (1983). The histogram routine utilizes the graphics set available on the CBM/PET/C-64 microcomputers.

An application of spectral analysis was given by Campbell and Shipp (1974); only brief details of the procedure follow. The first step is the removal of longterm trend from the sample values, which in this case is achieved by subtraction of a polynomial regression line from the data to give a series of difference values. For a sample of several hundred readings, a first- or seconddegree polynomial would normally be sufficient for subtraction, but if high spectral density persists at zero frequency, a higher order polynomial could be used. The nth-order regression and F-distribution routines used in this program to obtain the difference values are from Poole and Borchers (1979).

The author's mailing address is: Department of Biology, The University of Wollongong, P.O. Box 1144, Wollongong, N.S.W. 2500, Australia.
After computing the difference values for the sample, it is necessary to find the autocorrelation function. Autocorrelation is essentially the correlation of a sample moved on itself for $0,1,2 \ldots$ lags (unit time intervals). The autocorrelation value for any sample using zero lag is, of course, 1 . The series of autocorrelations is termed the sample autocorrelation function. Using this in place of the original sample for spectral analysis has the effect of canceling out noise but not the periodic components of the sample, particularly for samples of large size. Normally, $L<N / 2$ or $N / 3$, where $L$ is the number of lags and $\mathrm{N}$ is the sample size, is a sufficient length for the autocorrelation function.

The next step is to find the spectral density estimate. This is the Fourier cosine transform of the sample autocorrelation function. The existence of periodic components in the sample show as peaks in the spectral-density estimate. However, the spectral-density estimate often fluctuates widely, like a histogram with class intervals too narrow due to residual noise. This instability can be reduced by using the Tukey (or other) lag window to give a smoothed spectral-density estimate. Part of the technique of spectral analysis is to find a reasonable compromise between stability and fidelity, since information can be lost with excessive smoothing. The procedure followed to achieve this is termed window closing (see Jenkins \& Watts, 1968). Plotting the smoothed spectral-density estimate (the spectrogram) as a histogram on the screen simplifies the procedure of window closing. Using a small lag results in a smooth spectrum (i.e., the viewing window over which the spectrum is averaged is wide), but some details are likely to be masked. Increasing the lag size (i.e., narrowing the window) increases fidelity. This procedure can be continued until a satisfactory spectrogram is obtained. It means that several spectrograms will be viewed before a decision is made on which is the best window, or bandwidth, to use.

Operation. The program is menu based and simple to operate. The user is taken systematically by a series of prompts through the various steps of spectral analysis, as outlined above. The system is very flexible, and it is possible to backtrack through the program, which maximizes the ease of finding the most satisfactory spectrogram for a given set of data. The output can be for frequencies ranging from 0 to 0.5 , or for any specified period range in steps of 1 or .01 unit time intervals.

A worked sample illustrating the use of spectral analysis in identifying hidden periodicities in a synthetic time series was given by Campbell and Shipp (1974). The sample has 72 data values and consists of three periodicities $(20,10$, and 2.5$)$ and a noise component. An effective way of trying out the program is to follow this example through. The spectral-density values obtained will be slightly different from those recorded in the Campbell and Shipp (1974) paper. This is because they are calculated 
in this program on untruncated difference and autocorrelation values.

It is sometimes convenient to plot spectral density on a linear scale, as is done using this program (Broom, 1979; Chatfield \& Pepper, 1971). Using a logarithmic scale has the advantage that confidence intervals then have constant width for peaks at different spectral densities. If this is required, it is a simple matter to obtain a printout of the spectral-density values, plot them on a log scale, and add the bandwidth and the required confidence interval for the plot as described by Campbell and Shipp (1974) and Jenkins and Watts (1968).

Limitations. There are two main limitations of the program. First, a maximum sample size for a $32 \mathrm{~K}$ machine would be on the order of about 400 , depending on the number of autocorrelation lags and on the frequency and period intervals used. Second, the time required to run through a sample of several hundred is of the order of tens of minutes. One method of reducing the running time would be to use a compiler, such as "PETSPEED," but I have not tried this. For moderately sized samples, though, the program could be useful for a number of readers, particularly those who do not have access to a large mainframe computer and require an easy-to-operate system.

Availability. A listing of the program is available on request. A cassette tape of the program is available for $\$ 5.00$ to cover costs. The cassette tape format is compatible with all Commodore computers.

\section{REFERENCES}

BRoom, D. M. (1979). Methods of detecting and analysing activity rhythms. Biology of Behavior, 4, 3-18.

BRooM, D. M. (1980). Activity rhythms and position references of domestic chicks which can see a moving object. Animal Behaviour, 28, 201-211.
CAMPBELL, D. J. (1983). An editor and high-resolution histogram plotter for the CBM/PET microcomputer. Behavior Research Methods \& Instrumentation, 15, 618-619.

CAMPBELl, D. J., \& FOREST, J. (1984). The KIM-1 microcomputer as an eight-channel data logger. Behavior Research Methods, Instruments, \& Computers, 16, 315-319.

CAMPBELL, D. J., \& SHIPP, E. (1974). Spectral analysis of cyclic behaviour with examples from the field cricket Teleogryllus commodus (Walk.). Animal Behaviour, 22, 862-875.

CaMPBell, D. J., \& SHIPP, E. (1979). Regulation of spatial pattern in populations of the field cricket Teleogryllus commodus (Walker). Zeitschrift für Tierpsychologie, 51, 260-268.

Chatfield, C., \& Pepper, M. P. G. (1971). Time-series analysis: An example from geophysical data. Applied Statistics, 20, 217-238.

DAAN, S., \& SLOPSEMA, S. (1978). Short-term rhythms in foraging behaviour of the common vole, Microtus arvalis. Journal of Comparative Physiology, 127, 215-227.

Jenkins, G. M., \& WATTS, D. G. (1968). Spectral analysis and its applications. San Francisco: Holden-Day.

LiNKENS, D. A. (1979). Estimation of circadian rhythm components using auto-regressive modelling methods. Journal of Interdisciplinary Cycle Research, 10, 1-40.

MARTIN, P. H., \& UNwin, D. M. (1980). A microwave doppler radar activity monitor. Behavior Research Methods \& Instrumentation, 12, 517-520.

POOLE, L., \& Borchers, M. (1979). Some common basic programs (3rd ed.). Berkeley, CA: Osborne/McGraw-Hill.

RUSAK, B., \& ZUCKER, I. (1975). Biological rhythms and animal behavior. Annual Review of Psychology, 26, 137-171.

SAUNDERS, D. S. (1977). An introduction to biological rhythms. Glasgow: Blackie.

StuRgis, S. P. (1983). A spectral-analysis tutorial with examples in FORTRAN. Behavior Research Methods \& Instrumentation, 15, 377-386.

WESTMAN, R. S. (1977). Environmental languages and the functional bases of animal behavior. In B. A. Hazlett Quantitative methods in the study of animal behavior (pp. 145-201). New York: Academic Press.

(Manuscript accepted for publication July 26, 1984.) 